\title{
Performance of All-Optical XNOR Gate Based on Two-Photon Absorption in Semiconductor Optical Amplifiers
}

\begin{abstract}
Amer Kotb
Department of Physics, Faculty of Science, University of Fayoum, Fayoum 63514, Egypt

Correspondence should be addressed to Amer Kotb; amer_22003@yahoo.com

Received 12 September 2014; Revised 4 December 2014; Accepted 5 December 2014; Published 31 December 2014

Academic Editor: José Luís Santos

Copyright (C) 2014 Amer Kotb. This is an open access article distributed under the Creative Commons Attribution License, which permits unrestricted use, distribution, and reproduction in any medium, provided the original work is properly cited.

All-optical logic XNOR gate is realized by a series combination of XOR and INVERT gates. This Boolean function is realized by using Mach-Zehnder interferometers (MZIs) and exploiting the nonlinear effect of two-photon absorption (TPA) in semiconductor optical amplifiers (SOAs). The employed model takes into account the impact of amplified spontaneous emission (ASE), input pulse energy, pulsewidth, SOAs carrier lifetime, and linewidth enhancement factor ( $\alpha$-factor) on the gate's output quality factor ( $Q$-factor). The outcome of this study shows that the all-optical XNOR gate is indeed feasible with the proposed scheme at $250 \mathrm{~Gb} / \mathrm{s}$ with both logical correctness and acceptable quality.
\end{abstract}

\section{Introduction}

The development of all-optical logic technology is important for a wide range of applications in all optical networks, including high speed all-optical packet routing and optical encryption [1]. An important step in the development of all-optical logic technology, which includes key functionalities in fundamental and system-oriented level such as buffering, demultiplexing, clock recovery, packet processing, wavelength conversion, data regeneration, and optical encryption/decryption, is the demonstration of optical logic elements that can operate at ultrahigh speeds. Alloptical logic gates based on several different schemes have been demonstrated and reported, including that based on dual semiconductor optical amplifier (SOA) Mach-Zehnder interferometer (MZI) [2, 3], semiconductor laser amplifier loop mirror (SLALM) [4], ultrafast nonlinear interferometer (UNI) [5], four-wave mixing (FWM) process in SOA [6], or cross-gain modulation (XGM) or cross-phase modulation (XPM) in nonlinear devices [7]. All-optical logic XNOR gate using SOA is described and demonstrated earlier [813]. However the main limitation imposed on most of these schemes is that they cannot keep conveniently pace with the upgrades of single channel data rates in the effort to satisfy the unceasing bandwidth demand [14]. Thus in this work, we propose to address this critical issue by exploiting two-photon absorption (TPA) in SOAs, which are placed in the two arms of MZI operated in probe-dual pump mode. According to relevant pump-probe experiments, when a data modulated pump beam of appropriate intensity is launched into a SOA, the phase induced through TPA on a weak probe signal can change as fast as $1 \mathrm{ps}$, which subsequently can enable ultrafast interferometric switching of the same order [15]. Pump-probe experiments have shown that phase change takes place in duration $\sim 1 \mathrm{ps}$ or less when the pump and probe signals are injected into a SOA [16, 17]. Still so far previous reports on all-optical logic gates based on TPA process have not treated the XNOR gate [18-22]. However ASE is a degradation factor of the SOA response $[23,24]$ and should be considered in order to obtain realistic and accurate results for the intended all-optical logic at the pursued ultrafast data rate. In this work, I continue, extend, complete, and generalize the relevant previous work based on TPA [18-22] by investigating the high-speed performance of the all-optical logic XNOR gate with the help of numerical simulation conducted at a repetition rate of $\sim 250 \mathrm{~Gb} / \mathrm{s}$. Among these approaches, SOA is believed to be a key component for alloptical logic gates, because it has a stronger nonlinearity than optical fibers and it can be integrated more easily. However the speed of conventional bulk SOA operation is limited by 
the finite gain and phase recovery time of their temporal response. The study is carried out when the effect of the ASE is taken into account in the simulation analysis. The primary noise in this calculation (which lowers the quality factor ( $Q$ factor)) in the absence of ASE noise is pattern effects resulting from long recovery times of gain and gain induced phase change. The ASE causes additional output noise through spontaneous-spontaneous beat noise and signal-spontaneous beat noise. In addition if one were to measure the error rate of the gate output, the dark current of the photodiode, shot noise and thermal noise need to be considered. The ASE-related noise depends on the spontaneous emission factor $\left(N_{\mathrm{sp}}\right)$ of the amplifier [1]. The employed model takes into account the impact of pulse energy, pulsewidth, SOAs carrier lifetime, and linewidth enhancement factor ( $\alpha$-factor) on the gate's output $Q$-factor. The outcome of our study shows that the all-optical $\mathrm{XNOR}$ gate is indeed feasible with the proposed scheme at $250 \mathrm{~Gb} / \mathrm{s}$ with both logical correctness and acceptable quality. The input intensities are high enough so that the two-photon induced phase change is larger than the regular gain induced phase change. The advantage of this model is the lower power consumption and higher power transmission than in [18-22].

\section{SOA-MZI Model}

The operation of the SOA-MZI can be theoretically described by using a rate equation model as shown in [11-17].

The magnitude of the TPA-induced phase shift can be expressed as [18-22]

$$
\Phi_{\mathrm{TPA}}=-0.5 \beta \alpha_{\mathrm{TPA}} S(t) L,
$$

where $\beta$ is the TPA coefficient, $\alpha_{\text {TPA }}$ is the linewidth enhancement factor due to TPA process, and $L$ is the effective length of SOA active region. The negative sign represents the observation that the TPA induced phase change is in an opposite direction from that for the gain-induced phase change [10]. The experimentally derived $\alpha_{\mathrm{TPA}}$ is $\sim 4-5$ and the quantity $\beta$ is $\sim 20-35 \mathrm{~cm} / \mathrm{GW}[10]$. For a SOA having typical active region cross section $5 \times 10^{-9} \mathrm{~cm}^{2}$ and effective length $2 \mathrm{~mm}, \beta=30 \mathrm{~cm} / \mathrm{GW}$ and $\alpha_{\mathrm{TPA}}=4$. The peak phase change experienced by the probe would be around $0.4 \pi$. This phase change is large enough for temporal interference of probe pulses traveling through the two arms of the MZI.

The carrier density induced phase change is given by

$$
\Phi(t)=-0.5\left[\alpha h(t)+\alpha_{\mathrm{CH}} h_{\mathrm{CH}}(t)\right]-0.5 \beta \alpha_{\mathrm{TPA}} S(t) L,
$$

where $\alpha$ is the traditional linewidth enhancement factor and $\alpha_{\mathrm{CH}}$ is the carrier heating alpha factor.

The data inputs are assumed to be Gaussian-shaped pulse streams with temporal profile [18-24]:

$$
P_{\mathrm{A}, \mathrm{B}}(t)=\sum_{n=-\infty}^{n=+\infty} a_{n \mathrm{~A}, \mathrm{~B}} \frac{2 \sqrt{\ln (2)} P_{0}}{\sqrt{\pi} \tau_{\mathrm{FWHM}}} \operatorname{Exp}\left(-\frac{4 \ln (2)(t-n T)^{2}}{\tau_{\mathrm{FWHM}}^{2}}\right),
$$

where $a_{n \mathrm{~A}, \mathrm{~B}}$ represents the $n$th pulse in the data streams and can take the logical value " 1 " or " 0 " with equal probability, $P_{0}$ is the input pulse energy, $T$ is the bit period which is given by $1000 /$ data rate, and $\tau_{\text {FWHM }}$ is the pulsewidth (full width at half maximum).

\section{XNOR Model}

3.1. Operation Principle. In this study, XNOR gate is realized by a series combination of XOR and INVERT gates [8-10]. For XOR operation, two data signals A and B are injected into the SOAs incorporated in the upper and lower arms of MZI 1. A clock stream comprising continuous " 1 "s is introduced in the setup from the middle input port of the configuration, as shown in Figure 1. The data signals $A$ and $B$, which are spectrally located at wavelengths $\lambda_{1}$ and $\lambda_{3}$, respectively, induce via cross-phase modulation (XPM) a phase shift in the split components of the clock signal, at wavelength $\lambda_{2}$, in each SOA. Then the recombined clock signal at the output of MZI 1 carries the result of XOR operation between the binary content of data A and B. For proper signal discrimination wavelength $\lambda_{2}$ must be chosen so that it is different from $\lambda_{1}$ and $\lambda_{3}$, while $\lambda_{1}$ and $\lambda_{3}$ need not be different. Initially MZI 1 is balanced, and so when $A=$ " 0 " and $B=$ " 0 " the decomposed clock signal components traveling through the two MZI arms do not acquire any phase shift in the respective SOAs. Thus when they recombine at the output the result is "0." However when A = " 1 ", B = " 0 ", the clock signal replica traveling through the upper arm together with signal A acquires due to XPM a phase change, while its counterpart traveling through the lower arm does not suffer any such change. This results in " 1 " at the output. The same happens when $\mathrm{A}=$ " 0 ," $\mathrm{B}=$ " 1 ". However when $\mathrm{A}=$ " 1 " and $\mathrm{B}=$ " 1 " the phase changes induced on the clock signal constituents traveling through both MZI arms are equal, hence the output is " 0 ." The INVERT operation is obtained similarly to XOR operation if one of the data inputs is replaced by a clock signal. Then in order to realize the XNOR operation, the XOR output from MZI 1 is launched into the upper arm of MZI 2. Concurrently a continuous wave $(\mathrm{CW})$ beam and another clock signal are launched into the middle and bottom input ports, respectively, as shown in Figure 1. The effect of ASE on the performance of the XNOR gate is due to the contribution both from the XOR and INVERT operations.

3.2. Simulation. The modulated clock signal, which plays the role of the probe, interferes with the two arms of the MZIs obeying the following formula [24]:

$$
\begin{aligned}
P_{\text {out }}(t)=0.25 P_{C}\left\{G_{1}(t)+G_{2}(t)\right. & \\
& \left.-2 \sqrt{G_{1}(t) G_{2}(t)} \operatorname{Cos}\left[\Phi_{1}(t)-\Phi_{2}(t)\right]\right\},
\end{aligned}
$$

where $P_{C}$ is the input probe power, while $G_{1,2}(t)$ and $\Phi_{1,2}(t)$ are for the time-dependent gains and total phase shifts experienced by the copies of the $\mathrm{CW}$ signal. The instantaneous 


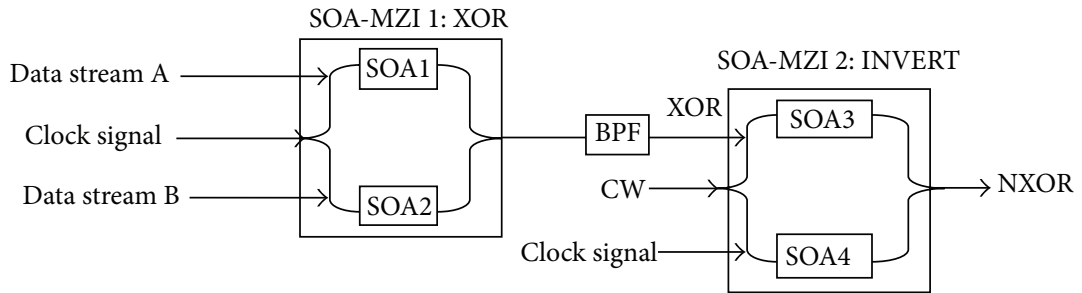

FIgURE 1: Schematic of a XNOR logic gate using two MZIs in series combination. BPF: band-pass filter.

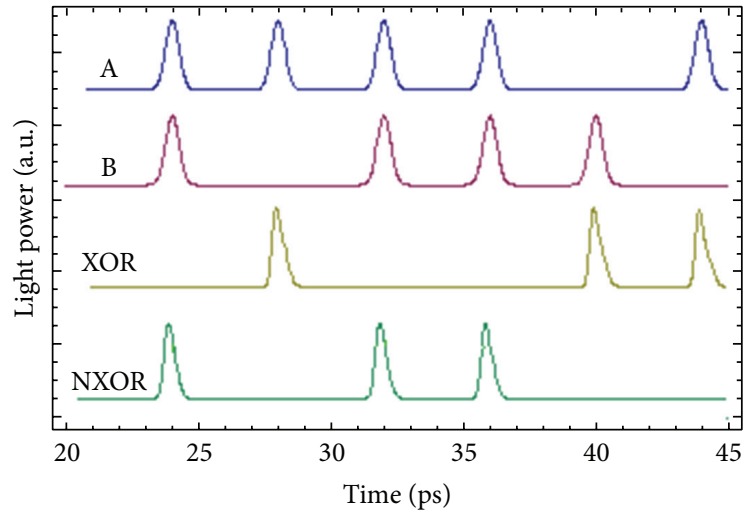

(a)

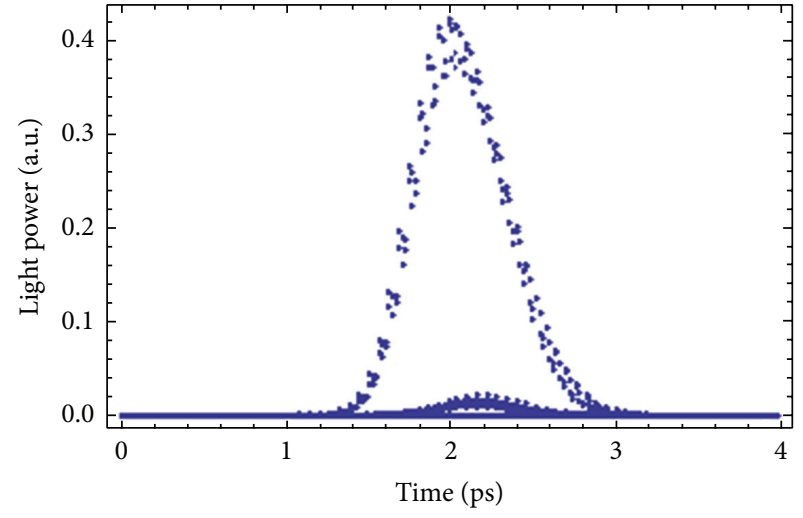

(b)

FIGURE 2: (a) Simulation results of SOAs-MZI for XNOR operation. Top two traces are the signals A and B; the third trace is the XOR output shown for logical comparison and the bottom trace is XNOR output; (b) eye diagram for XNOR.

optical intensity inside SOA-MZI1 for the XOR gate is given by

$$
\begin{aligned}
& P_{1}(t)=P_{\mathrm{A}}(t)+P_{\mathrm{C}}, \\
& P_{2}(t)=P_{\mathrm{C}}+P_{\mathrm{B}}(t) .
\end{aligned}
$$

The instantaneous optical intensity inside SOA-MZI2 for the INVERT gate is given by

$$
\begin{gathered}
P_{3}(t)=P_{\mathrm{XOR}}(t)+P_{\mathrm{CW}}, \\
P_{4}(t)=P_{\mathrm{CW}}+P_{\mathrm{C}} .
\end{gathered}
$$

The SOA parameters are defined and taken throughout the simulation as listed in Table 1.

Figure 2(a) illustrates the simulation results for the XNOR operation between an indicative pattern of data signal A and an indicative pattern of data signal B shown in the top two traces. The third trace shows for logical comparison the XOR output and the bottom trace shows the XNOR output after SOA-MZIs 1 and 2, respectively. The eye diagram that corresponds to XNOR operation is shown in Figure 2(b). In the absence of ASE noise the primary source of noise in the calculations, which lowers the $Q$-factor, is due to the pattern effects that result from long recovery times of gain and gain induced phase changes. The pulsewidth (full width at half maximum) is $0.5 \mathrm{ps}$, the data stream average power is $162 \mathrm{~mW}$, and the SOA active region cross section is $5 \times 10^{-9} \mathrm{~cm}^{2}$.

To investigate the "quality" of XNOR operation by simulation, the Q-factor of the XNOR output signal has been
TABLE 1: List of input signal and SOA parameters treated as fixed throughout the simulation analysis, by symbol, definition, and value.

\begin{tabular}{llc}
\hline Symbol & Definition & Value \\
\hline$G_{0}$ & SOAs unsaturated power gain & $30 \mathrm{~dB}$ \\
$P_{0}$ & Input pulse energy & $0.01 \mathrm{pJ}$ \\
$\tau_{\mathrm{FWHM}}$ & Pulsewidth & $0.5 \mathrm{ps}$ \\
$\tau_{c}$ & SOA carrier lifetime & $300 \mathrm{ps}$ \\
$\tau_{\mathrm{CH}}$ & Temperature relaxation rate & $0.3 \mathrm{ps}$ \\
$\tau_{\mathrm{SHB}}$ & Carrier-carrier scattering rate & $0.1 \mathrm{ps}$ \\
$\varepsilon_{\mathrm{CH}}$ & Carrier heating nonlinear gain & $0.08 \mathrm{~W}^{-1}$ \\
& suppression factor \\
$\varepsilon_{\mathrm{SHB}}$ & Spectral hole burning nonlinear gain & $0.08 \mathrm{~W}^{-1}$ \\
$\alpha$ & suppression factor & 5 \\
$\alpha_{\mathrm{CH}}$ & Traditional linewidth enhancement factor & 1 \\
$\alpha_{\mathrm{SHB}}$ & Carrier heating alpha factor & 0 \\
$P_{\text {sat }}$ & Spectral hole-burning alpha factor & $30 \mathrm{~mW}$ \\
$\alpha_{\mathrm{TPA}}$ & Saturation power & Linewidth enhancement factor due to \\
$\beta$ & TPA & TPA coefficient
\end{tabular}

calculated. This metric gives information for the optical signal-to-noise ratio in digital communications and is defined as $Q=\left(S_{1}-S_{0}\right) /\left(\sigma_{1}+\sigma_{2}\right)[18-24]$, where $S_{1}, S_{0}$ are the average intensities of the expected "1"s and "0"s and $\sigma_{1}, \sigma_{2}$ are 


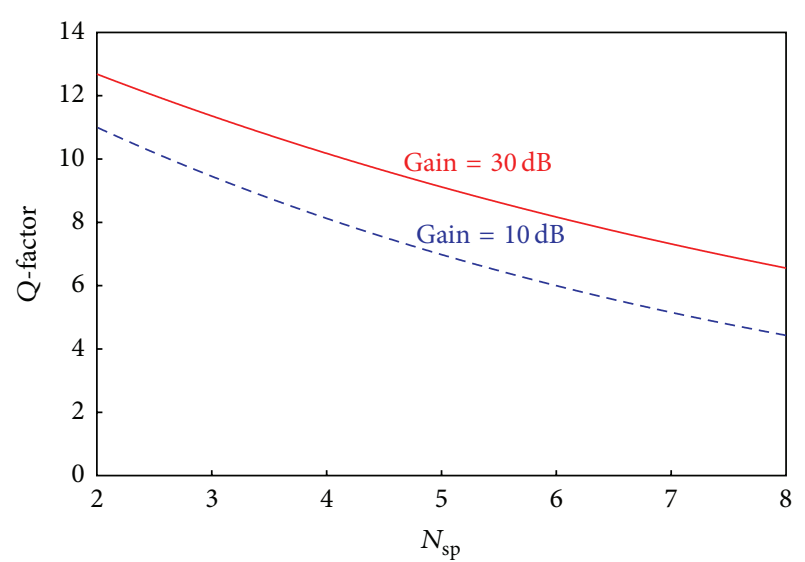

FIgURE 3: Q-factor versus $N_{\text {sp }}$ for XNOR operation.

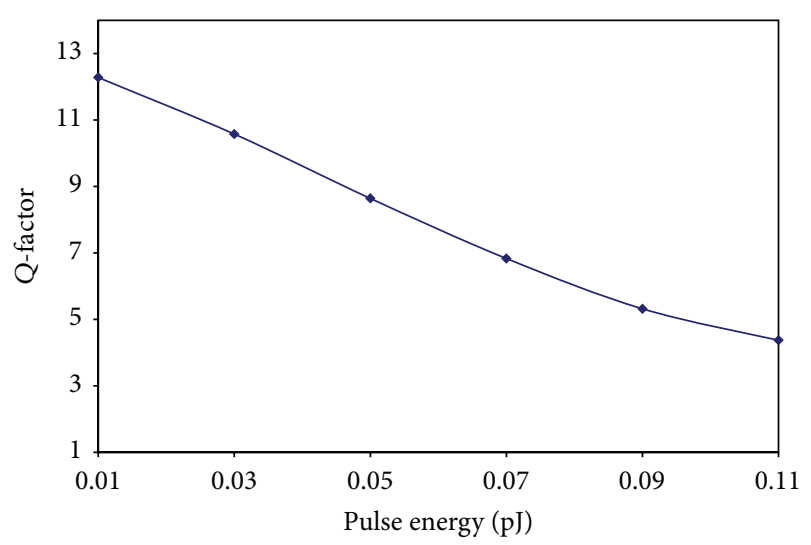

FIgURE 4: Q-factor versus input pulse energy for XNOR operation.

the standard deviations of those intensities [1]. I assume an optical amplifier with unity coupling efficiency, uniform gain $G$, over an optical bandwidth $B_{0}$, and an input power of $P_{\text {in }}$ at optical frequency $v$ centered in the optical pass bandwidth $B_{0}$. Then the ASE power in the optical bandwidth $B_{0}$ is related to $N_{\text {sp }}[23,24]$ :

$$
P_{\mathrm{ASE}}=N_{\mathrm{sp}}(G-1) h v B_{0},
$$

where $h$ is Plank's constant. The ASE noise is added numerically using (7) to the pattern effect noise to obtain the Qfactor. The $Q$-factor versus $N_{\text {sp }}$ for the XNOR output is shown in Figure 3. For an ideal amplifier $\left(N_{\mathrm{sp}}=2\right)$, the $Q$-factor is 12.34 at $250 \mathrm{~Gb} / \mathrm{s}$.

To obtain further information on the XNOR performance, the $Q$-factor versus input single-pulse energies is calculated as shown in Figure 4. An increase of input pulse energy will saturate the SOAs more easily, which results in a decrease of the $Q$-factor.

The $Q$-factor is also sensitive to the input pulsewidth and the SOA carrier lifetime as shown in Figure 5. The Qfactor decreases when increasing the pulsewidth because two neighboring pulses tend to overlap for wider pulsewidth as shown in Figure 5(a). Figure 5(b) shows the dependence of $Q$-factor on the SOA carrier lifetime. The results show that

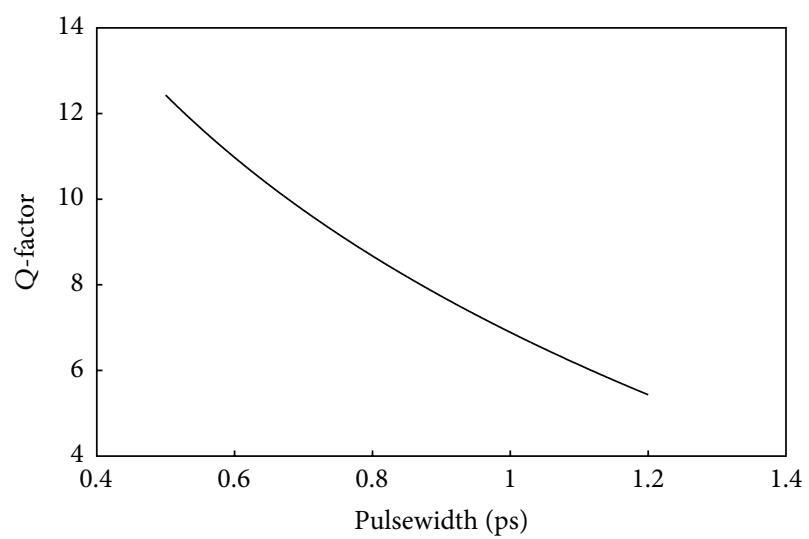

(a)

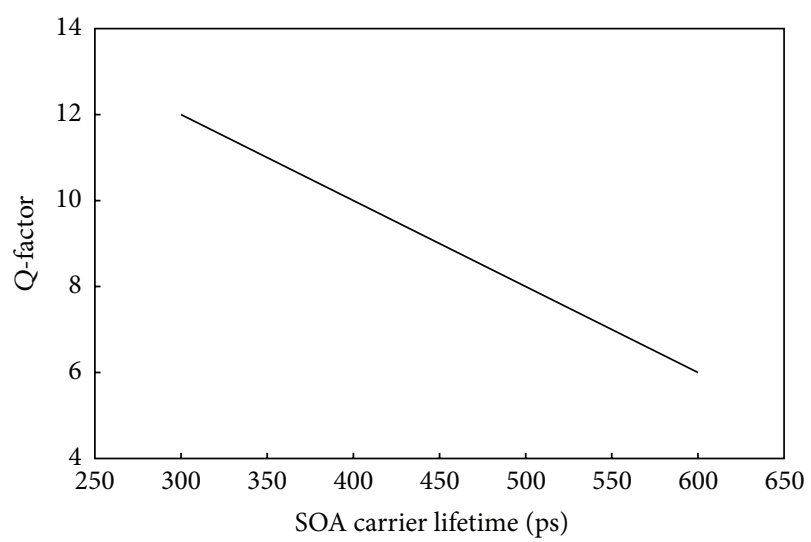

(b)

FIgURE 5: Calculated Q-factor as a function of (a) pulsewidth; (b) dependence of calculated $Q$-factor on SOA carrier lifetime for XNOR operation.

the $Q$-factor decreases as the SOA carrier lifetime increases. The SOA carrier lifetime determines the speed of gain and phase recovery in the active region, so the $Q$-factor is higher for a shorter carrier time.

The linewidth enhancement factor ( $\alpha$-factor) depends on the relative position of the amplifier gain peak and the signal wavelengths and thus can vary $Q$-factor as a function of $\alpha$ factor is shown in Figure 6. The $Q$-factor is larger for larger $\alpha$-factor because the phase changes are larger for large $\alpha$ factor and hence the XNOR signal (" 1 " values) is larger. This increases the signal-to-noise ratio and hence the $Q$-value.

\section{Conclusion}

In conclusion, the performance of all-optical logic XNOR gate based on two-photon absorption (TPA) using semiconductor optical amplifiers- (SOAs-) assisted Mach-Zehnder interferometers (MZIs) has been numerically simulated and investigated. The theoretical study has been carried out by solving the rate equations of the SOAs incorporated in the MZI arms when the effect of amplified spontaneous emission (ASE) is taken into account and included in the model through the spontaneous emission factor $\left(N_{\mathrm{sp}}\right)$. The impact 


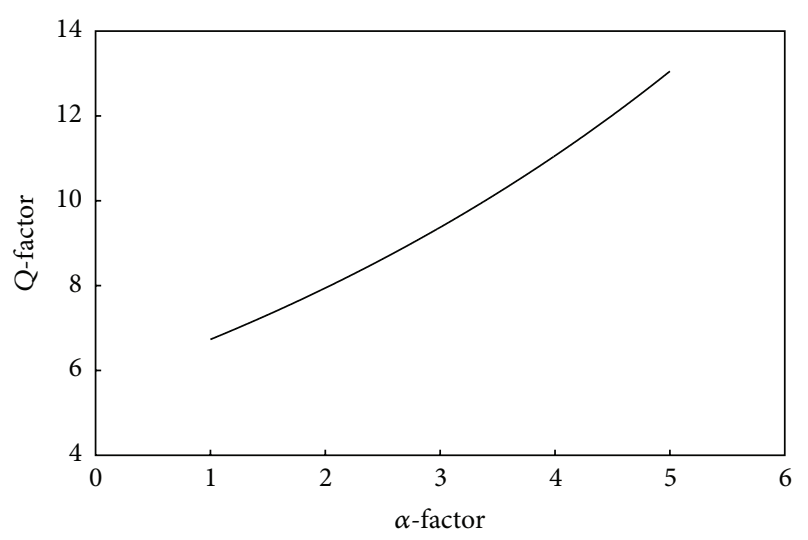

FIGURE 6: The simulated Q-factor dependence on linewidth enhancement factor ( $\alpha$-factor) for XNOR operation.

of ASE as well as of pulse energy, pulsewidth, SOAs carrier lifetime, and linewidth enhancement factor ( $\alpha$-factor) on the gate's output quality factor ( $Q$-factor) has been evaluated. The extracted results show that the treated logic gate is capable of operating at $250 \mathrm{~Gb} / \mathrm{s}$ with logical correctness and with the defined metric simultaneously being more than adequate. These results complement the suite of basic logic functions that can be implemented all-optically and at ultrafast data rates by exploiting the TPA nonlinear effect in SOAs. Finally, ASE effects are important and must be taken into account when all-optical logic gates are cascaded to form more complex circuits and subsystems of enhanced functionality.

\section{Conflict of Interests}

The author declares that there is no conflict of interests regarding the publication of this paper.

\section{References}

[1] N. K. Dutta and Q. Wang, Semiconductor Optical Amplifiers, World Scientific, New York, NY, USA, 2006.

[2] Q. Wang, G. Zhu, H. Chen et al., "Study of all-optical XOR using Mach-Zehnder interferometer and differential scheme," IEEE Journal of Quantum Electronics, vol. 40, no. 6, pp. 703-710, 2004.

[3] T. Fjelde, D. Wolfson, A. Kloch et al., "Demonstration of 20 Gbit/s all-optical logic XOR in integrated SOA-based interferometric wavelength converter," Electronics Letters, vol. 36, no. 22, pp. 1863-1864, 2000.

[4] T. Houbavlis and K. E. Zoiros, "Ultrafast pattern-operated alloptical Boolean XOR with semiconductor optical ampliferassisted Sagnac switch," Optical Engineering, vol. 42, no. 12, pp. 3415-3416, 2003.

[5] C. Bintjas, M. Kalyvas, G. Theophilopoulos et al., "20 Gbit/s alloptical with UNI gate," IEEE Photonics Technology Letters, vol. 12, p. 834, 2000.

[6] K. Chan, C.-K. Chan, L. K. Chen, and F. Tong, "Demonstration of $20-\mathrm{Gb} / \mathrm{s}$ all-optical XOR gate by four-wave mixing in semiconductor optical amplifier with RZ-DPSK modulated inputs," IEEE Photonics Technology Letters, vol. 16, no. 3, pp. 897-899, 2004.
[7] Z. Li, Y. Liu, S. Zhang et al., "All-optical logic gates using semiconductor optical amplifier assisted by optical filter," Electronics Letters, vol. 41, no. 25, pp. 51-52, 2005.

[8] E. Dimitriadou and K. E. Zoiros, "Proposal for ultrafast alloptical XNOR gate using single quantum-dot semiconductor optical amplifier-based Mach-Zehnder interferometer," Optics \& Laser Technology, vol. 45, no. 1, pp. 79-88, 2013.

[9] A. Kotb and K. E. Zoiros, "Simulation of all-optical logic XNOR gate based on quantum-dot semiconductor optical amplifiers with amplified spontaneous emission," Optical and Quantum Electronics, vol. 45, no. 11, pp. 1213-1221, 2013.

[10] A. Kotb and J. Maeda, "All-optical logic NXOR based on semiconductor optical amplifiers with the effect of amplified spontaneous emission," Optoelectronics Letters, vol. 8, no. 6, pp. 437-440, 2012.

[11] H. Sotoa, E. Alvareza, J. Topomondzob et al., "Design of an alloptical NOT XOR gate based on cross-polarization modulation in a semiconductor optical amplifier," Optics Communications, vol. 237, p. 121, 2004.

[12] S. Kumar and A. E. Willner, "Simultaneous four-wave mixing and cross-gain modulation for implementing an all-optical XNOR logic gate using a single SOA," Optics Express, vol. 14, no. 12, pp. 5092-5097, 2006.

[13] E. Dimitriadou and K. E. Zoiros, "All-optical XNOR gate using quantum-dot semiconductor optical amplifier-based MachZehnder interferometer," in Proceedings of the 8th International Symposium on Communication Systems, Networks and Digital Signal Processing (CSNDSP '12), pp. 1-5, July 2012.

[14] A. Bogoni, L. Potì, P. Ghelfi et al., "OTDM-based optical communications networks at $160 \mathrm{Gbit} / \mathrm{s}$ and beyond," Optical Fiber Technology, vol. 13, no. 1, pp. 1-12, 2007.

[15] H. J. S. Dorren, X. Yang, A. K. Mishra et al., "All-optical logic based on ultrafast gain and index dynamics in a semiconductor optical amplifier," IEEE Journal on Selected Topics in Quantum Electronics, vol. 10, no. 5, pp. 1079-1092, 2004.

[16] H. J. S. Dorren, G. D. Khoe, and D. Lenstra, "All-optical switching of an ultrashort pulse using a semiconductor optical amplifier in a Sagnac-interferometric arrangement," Optics Communications, vol. 205, no. 4-6, pp. 247-252, 2002.

[17] H. J. S. Dorren, X. Yang, D. Lenstra et al., "Ultrafast refractiveindex dynamics in a multiquantum-well semiconductor optical amplifier," IEEE Photonics Technology Letters, vol. 15, no. 6, pp. 792-794, 2003.

[18] A. Kotb, S. Ma, Z. Chen, N. K. Dutta, and G. Said, "All optical logic NAND gate based on two-photon absorption in semiconductor optical amplifiers," Optics Communications, vol. 283, no. 23, pp. 4707-4712, 2010.

[19] S. Ma, A. Kotb, Z. Chen, and N. K. Dutta, "All optical logic gates based on two-photon absorption," in Photonics North 2010, vol. 7750 of Proceedings of SPIE, Niagara Falls, Canada, September 2010.

[20] A. Kotb, "AND gate based on two-photon absorption in semiconductor optical amplifier," Optoelectronics Letters, vol. 9, no. 3, pp. 181-184, 2013.

[21] A. Kotb and K. E. Zoiros, "Performance of all-optical XOR gate based on two-photon absorption in semiconductor optical amplifier-assisted Mach-Zehnder interferometer with effect of amplified spontaneous emission," Optical and Quantum Electronics, vol. 46, no. 7, pp. 935-944, 2014.

[22] S. Ma, Z. Chen, and N. K. Dutta, "All-optical logic gates based on two-photon absorption in semiconductor optical amplifiers," Optics Communications, vol. 282, no. 23, pp. 4508-4512, 2009. 
[23] A. Kotb, S. Ma, Z. Chen, N. K. Dutta, and G. Said, "Effect of amplified spontaneous emission on semiconductor optical amplifier based all-optical logic," Optics Communications, vol. 284, no. 24, pp. 5798-5803, 2011.

[24] A. Kotb, All-Optical Logic Gates Using Semiconductor Optical Amplifiers, Lambert Academic Publishing, 2012. 

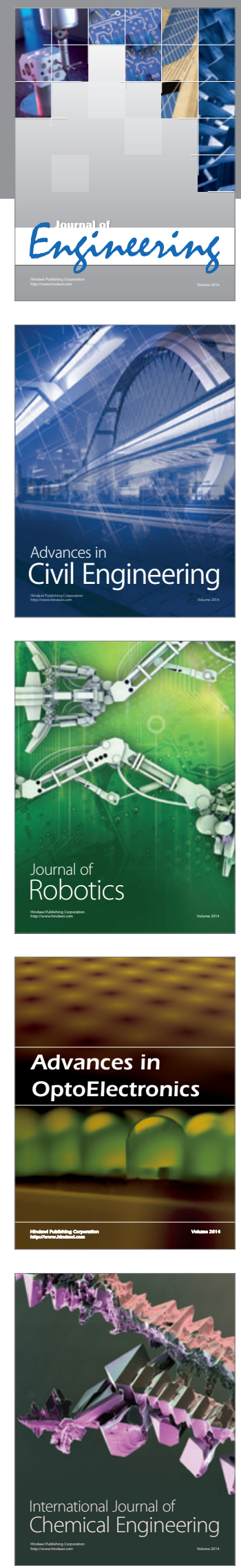

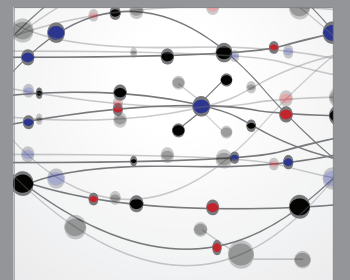

The Scientific World Journal
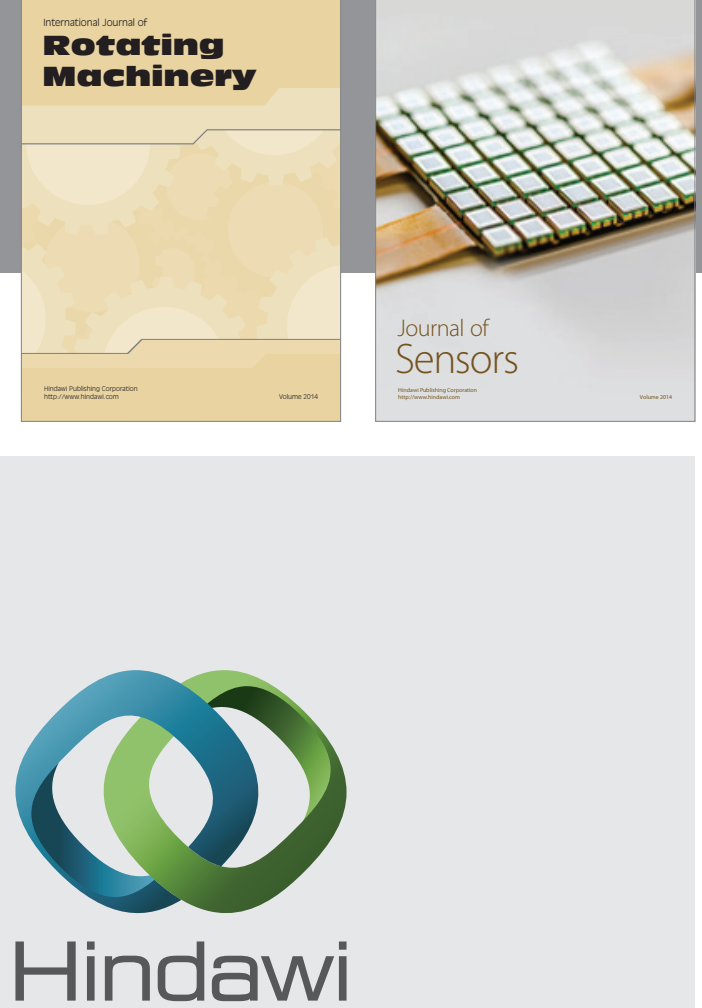

Submit your manuscripts at http://www.hindawi.com
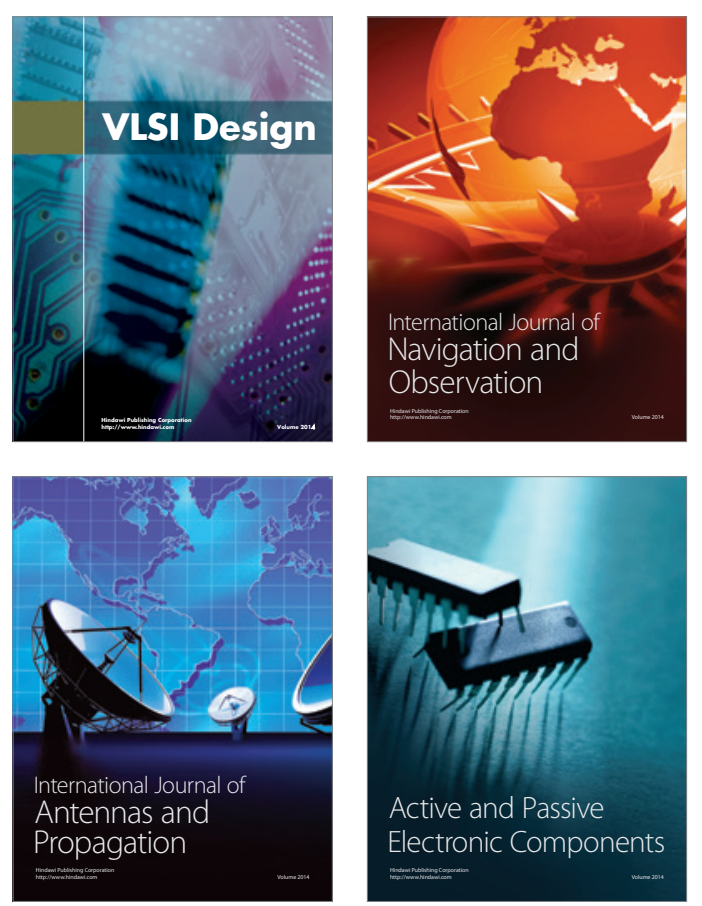
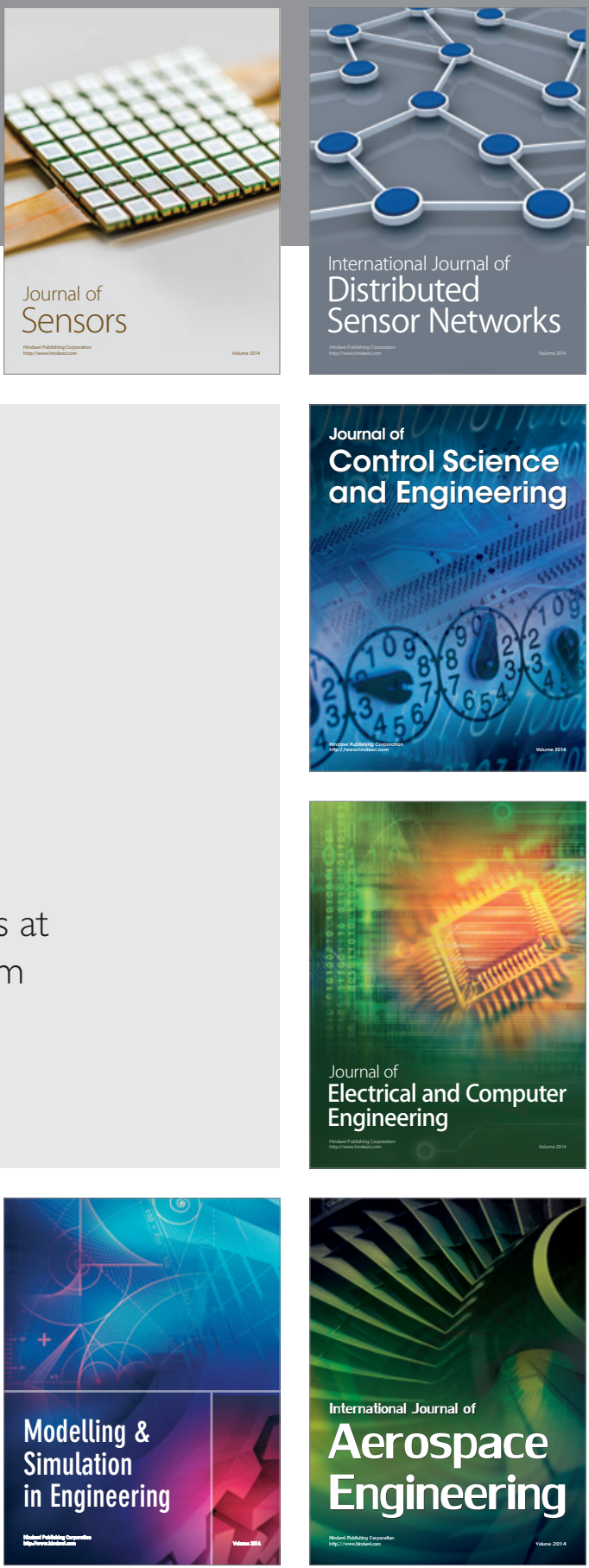

Journal of

Control Science

and Engineering
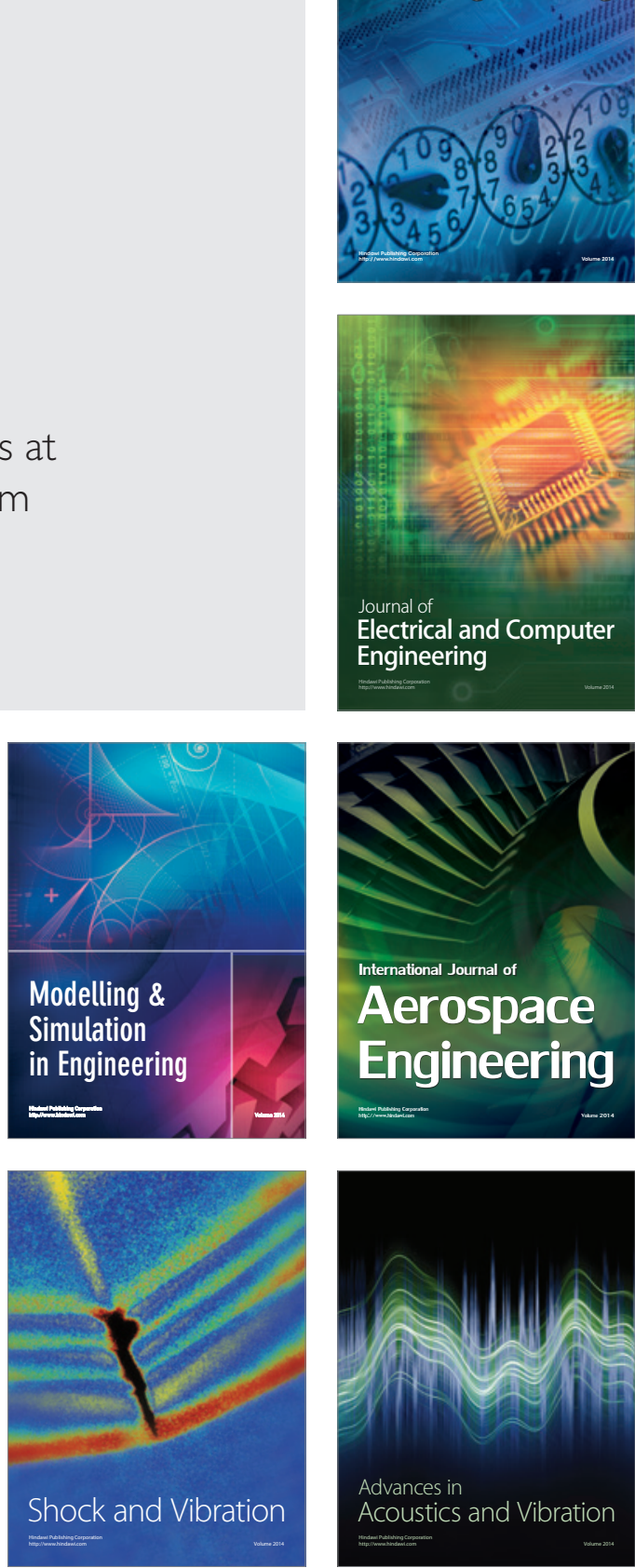